\title{
Congenital syphilis and glomerulonephritis with evidence for immune pathogenesis
}

\author{
J. WIGGELINKHUIZEN, R. O. C. KASCHULA, C. J. UYS, R. H. KUIJTEN, * and J. DALE \\ From the Red Cross War Memorial Children's Hospital, Rondebosch, Cape, and the Departments of Paediatrics and \\ Pathology, University of Cape Town, South Africa
}

\begin{abstract}
Wiggelinkhuizen, J., Kaschula, R. O. C., Uys, C. J., Kuijten, R. H., and Dale, J. (1973). Archives of Disease in Childhood, 48, 375. Congenital syphilis and glomerulonephritis with evidence for immune pathogenesis. In 3 infants with congenital syphilis the dominant clinical manifestation of syphilitic kidney disease was the nephrotic syndrome. Mesangioendothelial proliferation was present in 2 cases and mixed proliferative glomerulonephritis with crescent formation in the third. The severity of the clinical and histopathological abnormalities could be related to the apparent duration of the illness.

In all 3 cases immune complex deposition could be shown within and along the epithelial aspect of the glomerular basement membrane on light, electron, and immunofluorescent microscopy. These features, together with a reduced total serum haemolytic complement, suggest an immune pathogenesis of the glomerulonephritis associated with early congenital syphilis.
\end{abstract}

Congenital syphilis, though uncommonly associated with kidney disease, is nevertheless a welldocumented cause of the nephrotic syndrome in young infants. We present the clinical, laboratory, histopathological, electron microscopy, and immunofluorescent findings of the nephropathy before, during, and after treatment of early congenital syphilis. Evidence suggestive of an immune pathogenesis for the nephropathy of acquired syphilis was recently published (Braunstein et al., 1970; Bhorade et al., 1971). Our findings suggest a similar immunopathogenetic mechanism for the renal lesions of congenital syphilis.

\section{Case reports}

Case 1. A coloured girl (mixed race) aged 4 months was admitted for oedema of 2 months' duration. Pregnancy and delivery had been uneventful but the mother had received no antenatal care. No immunizations had been given. The infant had gross generalized oedema with ascites, a healing erythematous circinate rash on the trunk, thin sparse hair, anaemia, and $3 \mathrm{~cm}$ hepatosplenomegaly.

Pertinent laboratory findings are given in Table I. $X$-rays of the long bones revealed syphilitic periostitis and osteochondritis. An intravenous pyelogram was normal.

\footnotetext{
Received 14 August 1972.

*Present address: Wilhelmina Children's Hospital, Utrecht, The Netherlands.
}

The infant was treated with diuretics, blood transfusion, intravenous salt-free human albumin, and intramuscular procaine penicillin 200,000 units daily. Open renal biopsy was performed on the fourth day of penicillin therapy. Severe proteinuria and intermittent oedema persisted despite a further 3.3 megaunits procaine penicillin over 11 days and subsequent synthetic ACTH administration (Synacthen, Ciba, $0.25 \mathrm{mg}$ i.m.).

She was readmitted at 10 months for investigation of the persisting proteinuria. Clinical examination was normal. Pertinent laboratory findings-blood urea $56 \mathrm{mg} / 100 \mathrm{ml}$, serum creatinine $0.5 \mathrm{mg} / 100 \mathrm{ml}$, serum albumin $2.9 \mathrm{~g} / 100 \mathrm{ml}$, urine microscopy normal, urinary protein concentration $170 \mathrm{mg} / 100 \mathrm{ml}$. A second renal biopsy was performed to reassess the histopathological lesions, and a third course of procaine penicillin totalling $5 \cdot 1$ megaunits was then administered.

At the age of 16 months she was well but remained underweight $(8 \cdot 54 \mathrm{~kg})$. Laboratory findings were normal except for mild continuing proteinuria $(55 \mathrm{mg} / 100 \mathrm{ml})$.

Biopsy findings. Light microscopy: most glomeruli were enlarged with mesangioendothelial proliferation, polymorph exudation, capsular adhesions, and frequent epithelial crescents. Methenamine silver stains showed irregular thickening of the glomerular basement membrane with occasional 'spikes' on the epithelial aspect. The interstitium showed mild infiltration with polymorphs and plasma cells.

Repeat biopsy 6 months later showed that the severely affected glomeruli (more than $50 \%$ ) had been hyalinized, while many of the remaining functional 
TABLE I

Laboratory findings in 3 cases of congenital syphilis with glomerulonephritis

\begin{tabular}{|c|c|c|c|}
\hline & Case 1 & Case 2 & Case 3 \\
\hline $\begin{array}{l}\text { Urine microscopy } \\
\text { RBC } \\
\text { WBC } \\
\text { Casts } \\
\text { Urine protein }(\mathrm{mg} / 100 \mathrm{ml}) \\
\text { Serum proteins }(\mathrm{g} / 100 \mathrm{ml}) \\
\text { Albumin } \\
\alpha_{1}-\text { globulin } \\
\alpha_{2}-\text { globulin } \\
\beta \text {-globulin } \\
\gamma \text {-globulin } \\
\text { Ig immunoelectrophoresis } \\
\text { semiquantitative or } \\
\text { mg/100 ml } \\
\text { Blood urea (mg/100 ml) } \\
\text { Serum creatinine (mg/100 ml) } \\
\text { Serum cholesterol (mg/100 ml) } \\
\text { ASO titre (IU) } \\
\text { Total serum haemolytic complement (normal 160-210 C'H50 units/ml) } \\
\text { WR, VDRL, FTA-ABS tests }\end{array}$ & $\begin{array}{c}+++ \\
++ \\
++ \\
390-1000 \\
1 \cdot 7 \\
0 \cdot 3 \\
1 \cdot 4 \\
0 \cdot 6 \\
1 \cdot 0 \\
\text { IgG normal } \\
\text { IgA normal } \\
\text { IgM markedly raised } \\
91 \\
1 \cdot 4 \\
301 \\
200 \\
106 \\
\text { Positive }\end{array}$ & $\begin{array}{c}++ \\
+ \\
++ \\
350-420 \\
\\
1 \cdot 3 \\
0 \cdot 3 \\
0 \cdot 5 \\
0 \cdot 6 \\
1 \cdot 4 \\
755 \\
15 \\
650 \\
50 \\
0 \cdot 4 \\
163 \\
125 \\
\text { Very low } \\
\text { Positive }\end{array}$ & $\begin{array}{c}0 \\
0 \\
++ \\
260-990 \\
1 \cdot 0 \\
0 \cdot 3 \\
1 \cdot 2 \\
0 \cdot 6 \\
0 \cdot 3 \\
969^{\star} \\
103^{\star} \\
256^{\star} \\
61 \\
0 \cdot 4 \\
278 \\
125 \\
182 \\
\text { Positive }\end{array}$ \\
\hline
\end{tabular}

*In remission after treatment.

†Fluorescent treponema antibody absorption.

glomeruli still showed mild mesangioendothelial proliferation with capsular adhesions (Fig. 1).

Electron microscopy: the glomerular basement membrane showed irregular thickening with numerous electron-dense hump-like deposits mostly along the subepithelial aspect. The epithelial cell foot processes were fused and showed dense granularity in the neighbourhood of the deposits (Fig. 5). Villous hyperplasia of the epithelial cells, crescents, and mesangial proliferation were prominent.

The second biopsy showed advanced hyalinization. Identifiable glomerular basement membrane showed gross thickening with incorporation of the electrondense deposits.

Immunofluorescence of the second biopsy: in nonhyalinized glomeruli finely granular immunofluorescence

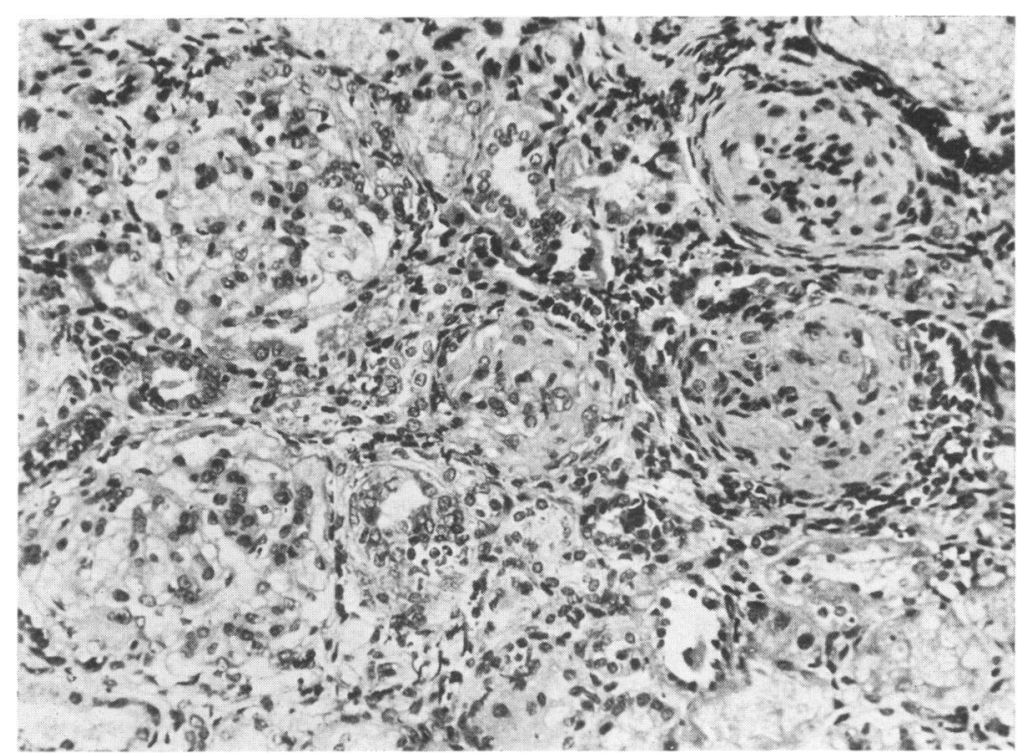

FIG. 1.-Case 1, second biopsy. Glomerular hyalinization. Remaining glomeruli are hypercellular and show capsular adhesions. (H. and E. $\times 133$.) 
TABLE II

Immunofluorescence studies ${ }^{\star}$ in renal biopsy specimens

\begin{tabular}{|c|c|c|c|c|c|c|}
\hline & & Intensity & & & Distribution & \\
\hline Case & 1 & 2 & 3 & 1 & 2 & 3 \\
\hline $\begin{array}{l}\text { Anti-IgG } \\
\text { Anti-IgA } \\
\text { Anti-IgM } \\
\text { Anti-C' } \\
\text { Antifibrinogen }\end{array}$ & $\begin{array}{r}++ \\
\pm \\
++ \\
++ \\
\pm\end{array}$ & $\begin{array}{r}++ \\
+ \\
+ \\
++ \\
+\end{array}$ & $\begin{array}{r}++t \\
++ \\
+ \\
++ \\
\pm\end{array}$ & $\begin{array}{r}+++ \\
+ \\
+++ \\
+++ \\
+++\end{array}$ & $\begin{array}{l}+++ \\
+++ \\
+++ \\
+++ \\
++\end{array}$ & $\begin{array}{r}+++ \\
+++ \\
++ \\
+++ \\
++\end{array}$ \\
\hline
\end{tabular}

$\star$ Brentjens et al., 1969.

Note: Intensity is graded from 0 to +++ . Distribution is graded from an occasional deposit (trace) to diffusely along the glomerular basement membrane $(+t+)$.

of moderate intensity was present along the outer aspect of and within the glomerular basement membrane. This was most marked with anti-IgG, anti-IgM, and anticomplement (Table II). In the hyalinized glomeruli some immunofluorescence could occasionally be seen with anti-IgM and anticomplement sera, but not with anti-IgG. Fluorescence in these glomeruli could not clearly be located along the basement membrane, but seemed to involve the mesangium and was of a patchy character.

Case 2. A 2-week-old African boy was admitted with abdominal distension and bilateral inguinal herniae. Pregnancy and delivery had been uneventful. Progressive generalized oedema and ascites developed in hospital. The infant was anaemic, had a $3 \mathrm{~cm}$ enlargement of liver and spleen, and palpably enlarged kidneys.

Pertinent laboratory findings are given in Table $\mathrm{I}$.
$X$-rays of the long bones revealed the bone lesions of congenital syphilis. An intravenous pyelogram was normal.

The infant was given intramuscular procaine penicillin 300,000 units daily and a blood transfusion. The oedema and ascites resolved within 2 weeks, but severe proteinuria persisted. Open renal biopsy was performed 10 days after penicillin therapy. He was discharged at the age of 2 months, oedema-free, but with persisting moderate proteinuria. Clinical examination and laboratory investigations were normal when seen at 5 months.

Biopsy findings. Light microscopy: there was diffuse enlargement of glomeruli with mesangioendothelial proliferation and mild polymorph exudation. Capsular adhesions but no crescents were seen (Fig. 2). Methenamine silver stains showed irregular thickening with 'spiking' on the epithelial surface of the glomerular basement membrane. The interstitium showed a moderate

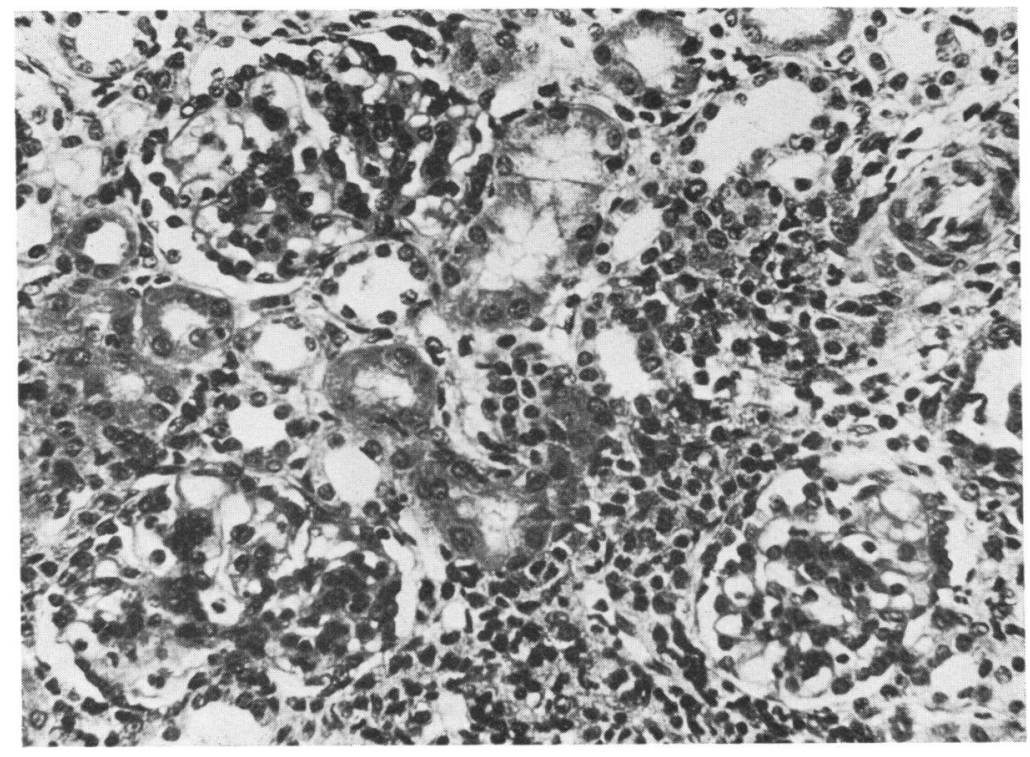

FIG. 2.-Case 2. Glomeruli show mesangioendothelial proliferation with capsular adhesions. The interstitium is infiltrated with mononuclear inflammatory cells. (H. and $E . \times 133$. 


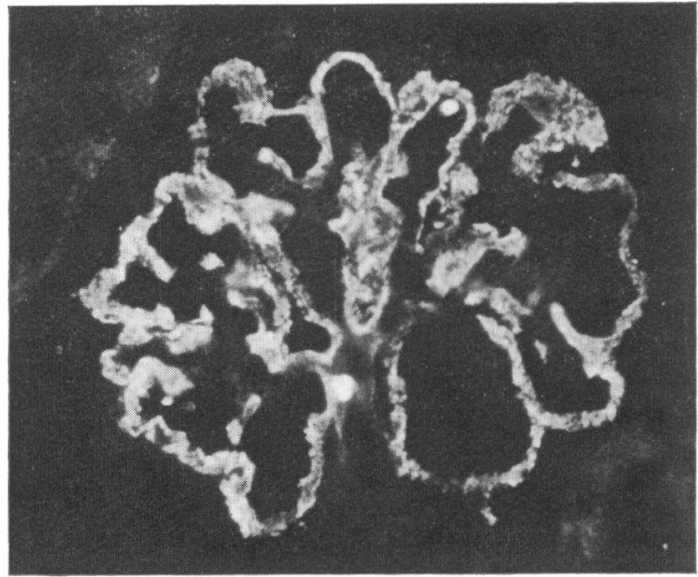

FIG. 3.-Case 2. Immunofluorescence with anti-IgG. $(\times 137$.

infiltrate of plasma cells, lymphocytes, and polymorphonuclear leucocytes.

Electron microscopy: numerous electron-dense deposits were seen within and particularly along the epithelial aspect of the glomerular basement membrane. Mesangial cells and matrix were increased.

Immunofluorescent microscopy: all glomeruli showed finely granular immunofluorescence of marked intensity diffusely along the outer aspect of and within the base- ment membrane (Fig. 3). It was most marked with anti-IgG and anticomplement, less so with anti-IgA and anti-IgM, and minimal with antifibrinogen (Table II).

Case 3. A coloured boy aged 2 months was admitted with gastroenteritis. Gross generalized oedema with ascites developed in hospital. The clinical, serological, and $x$-ray findings resembled the features of Cases 1 and 2. Pertinent laboratory findings are given in Table I.

The infant was treated with diuretics and a blood transfusion. Open renal biopsy was performed before the administration of penicillin. Within 4 weeks of the start of penicillin therapy $(6.3$ megaunits) the infant had improved, with loss of oedema and minimal residual proteinuria.

Renal biopsy was repeated 3 months after the first.

Biopsy findings. First biopsy (pretreatment): under light microscopy the ultrastructure (Fig. 4) and immunofluorescence showed features similar to those found in Case 2.

Second biopsy: under light microscopy the glomeruli showed mild mesangioendothelial hypercellularity while methenamine silver stains still revealed 'spikes' on the epithelial aspect of the glomerular basement membrane.

Electron microscopy: there was obvious diminution in the number of subepithelial and intramembranous deposits, many of which now had an unusual coarse particulate appearance under very high magnification.

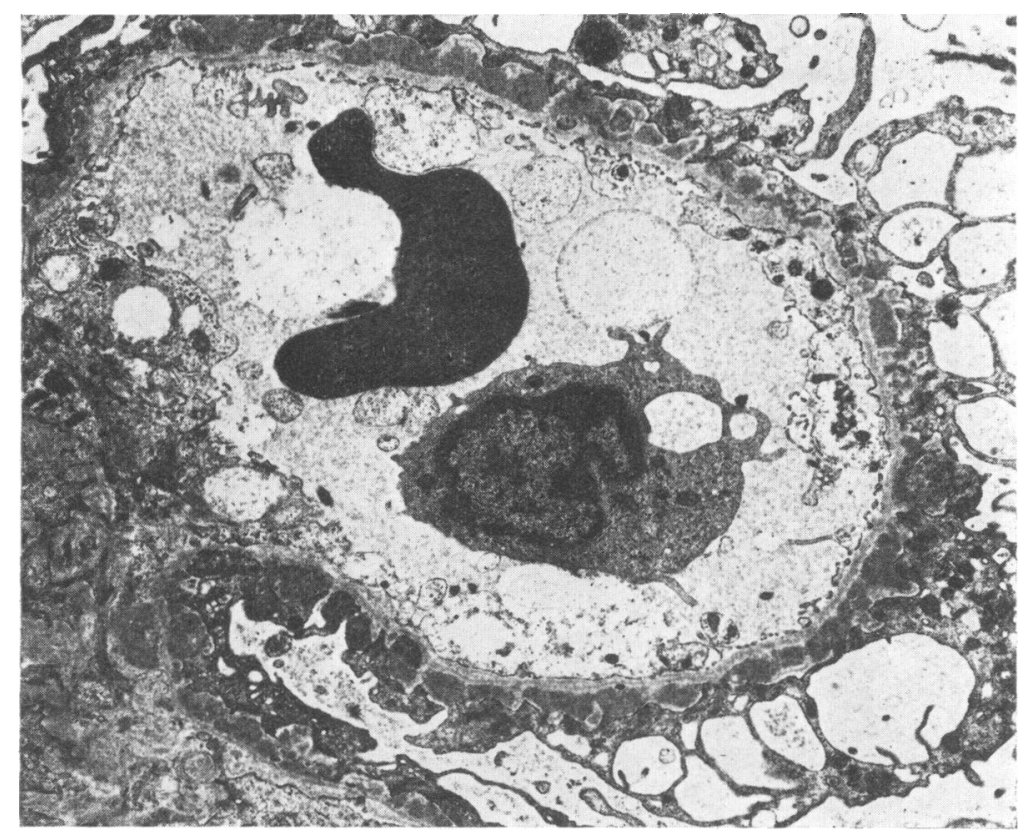

FIG. 4.-Case 3, first biopsy. Electron micrograph of glomerular capillary containing a polymorphonuclear leucocyte and an erythrocyte. There are numerous electron-dense subepithelial deposits along the basement membrane. The epithelial foot processes are fused and there is much vacuolation of the epithelial cell cytoplasm. $(\times 4800$. 




FIG. 5.-Case 1, first biopsy. Subepithelial electron-dense deposits in glomerular basement membrane, similar to those seen in Fig. 4, but shown here in higher magnification. $(\times 10312$.

\section{Discussion}

Hepatosplenomegaly, snuffles, sparse hair, skin lesions, anaemia, and thrombocytopenia suggested the diagnosis of congenital syphilis. This was confirmed by the positive serological tests on the infants and their mothers, and the $x$-ray findings of the bone lesions of early congenital syphilis.

The infants had features of severe renal disease with generalized oedema, ascites, massive proteinuria, hypoproteinaemia, and raised blood urea. One infant had macroscopical haematuria and another significant microhaematuria (Table I). Causes of renal disease other than syphilis were not apparent.

Penicillin therapy resulted in slow resolution of the clinical manifestations of congenital syphilis and healing of the histopathological lesions in the kidney. Herxheimer reactions were not encountered.

The clinical manifestations in our cases were similar to previous descriptions (Herrmann and Marr, 1935; Yampolsky and Mullins, 1945; Scully and Yamazaki, 1949; Mitchell, 1951; Taitz, Isaacson, and Stein, 1961 ; Papaioannou, Asrow, and Schuckmell, 1961; Pollner, 1966; McDonald, Wiggelinkhuizen, and Kaschula, 1971). Oedema is the most prominent clinical feature, though a nephritic element with haematuria and uraemia is common.

The serum protein electrophoretic pattern is characteristic of the nephrotic syndrome, though $\gamma$-globulin may be normal or even raised due to markedly raised IgM levels. The latter finding in the early weeks of life denotes intrauterine infection.
Urinary protein electrophoresis in 2 of the cases revealed a nonselective proteinuria.

The published histopathological features have varied from 'minimal changes' (Yuceoglu et al., 1966), membranoproliferative glomerulonephritis (McDonald et al., 1971), membranous glomerulonephritis (Pollner, 1966), to mixed endothelial and epithelial proliferation with crescent formation (Yampolsky and Mullins, 1945; Taitz et al., 1961).

In the first infant, who had macrohaematuria and moderate uraemia with the nephrotic syndrome, biopsy showed severe mixed proliferative glomerulonephritis with crescent formation and glomerular basement membrane thickening. The duration of symptoms and thus presumed exposure to the injurious agent was prolonged in this infant, and we suggest that this had a bearing on the severity of the inflammatory reaction in the glomeruli. Repeat biopsy 6 months later revealed scarring with glomerulosclerosis of more than half of the glomeruli (Fig. 1).

The other two infants had been ill for less than 2 weeks; both showed reversible mesangioendothelial proliferation with glomerular basement membrane thickening (Fig. 2), a lesion similar to that seen in the nephropathy of acquired syphilis (Braunstein et al., 1970; Bhorade et al., 1971; Brophy et al., 1964).

The tubules showed no gross abnormalities and interstitial infiltration with inflammatory cells was not prominent.

It is evident that resolution of the microscopical abnormalities, even if reversible, is slower than the clinical improvement.

Pathogenesis. Direct injury during the spirochataemic phase, antisyphilitic therapy with heavy metals and penicillin, increased susceptibility to poststreptococcal glomerulonephritis, hypersensititivity, and antigen-antibody reactions have been suggested as possible pathogenetic mechanisms.

On the basis of electron microscopy findings, Falls et al. (1965) first suggested an immune pathogenesis for the nephropathy of acquired syphilis. This was recently corroborated by the studies of Braunstein et al. (1970) and Bhorade et al. (1971).

The evidence presented suggests that the nephropathy of congenital syphilis similarly is an immune complex glomerulonephritis.

Light microscopy revealed glomerular injury with mesangioendothelial proliferation, epithelial crescents, and thickening of the glomerular basement membrane due to nonargyrophilic subepithelial deposits. On electron microscopy discrete electrondense deposits suggestive of immune complexes 
were seen in subepithelial positions along the glomerular basement membrane. On immunofluorescent staining, immunoglobulins and complement were shown in similar sites.

Braunstein et al. (1970), in their case of acquired syphilitic nephropathy, were able to show selective deposition of $\gamma-G_{2}$ heavy chains and $\gamma$-light chains, but complement was not found, and neither they nor Bhorade et al. (1971) were able to show antitreponemal activity of the deposited $\gamma$-globulin fractions. Bhorade et al. (1971) showed complement in the deposits on immunofluorescence, though serum complement was normal. We found a reduced total serum haemolytic complement in 2 infants and complement was shown in the immune complex deposits in the glomeruli in all 3 cases. The serum complement levels returned to normal after treatment with penicillin.

Damage to, and interference with, the function of the glomerulus occurs as a result of the inflammatory reaction induced by soluble antigenantibody complexes trapped in the glomerular basement membrane. This is mediated by the complement cascade, polymorphonuclear leucocytes, the coagulation system, and probably other mechanisms (Dixon, 1968; Dixon and Cochrane, 1970; McCluskey, 1970; Lewis and Couser, 1971).

The clinical and morphological resemblance between human immune complex-induced glomerulonephritis and experimental serum sickness glomerulonephritis in rabbits suggests similar pathogenic mechanisms. Experimentally, the earliest histopathological lesion is membranous glomerulonephritis, but as the experiment is continued, proliferative and progressive glomerulonephritis with scarring occurs (Germuth, Senterfit, and Pollack, 1967).

Congenital syphilis acquired during intrąuterine life may be present for weeks or months before diagnosis. Development of immune complex glomerulonephritis may be dependent, as under experimental conditions, on the degree of antigenic exposure and antibody response. The morphological lesions in our 3 cases correlated with the apparent duration of antigenic exposure.

Autologous IgG production in the fetus is limited, and it is interesting to speculate that, in the neonate, maternal IgG transmitted across the placental barrier participates in the formation of immune complexes.

Eradication of the antigen may be expected to be followed by clinical and histological cure, provided that the glomeruli have not sustained irreversible damage. Reversibility is probably related to the type, severity, and duration of the initial insult and to the presence or absence of a mechanism for perpetuating the disease process. Prolonged exposure to the injurious agent appears to result in severe and irreversible glomerular damage.

Treatment and prognosis. Congenital syphilis is preventable by good antenatal care. Serological tests for syphilis on umbilical cord blood will assure an early diagnosis postnatally. The IgM fluorescent treponemal antibody absorption test (IgM-FTA-ABS) appears reliable in the differentiation of passively transferred maternal antibody from that produced by the fetus in response to syphilitic infection.

Recommended treatment is either an intramuscular injection of benzathine penicillin G 100,000 units/kg which should be repeated 1 to 2 weeks later, or procaine penicillin $G 100,000$ units/kg per day for 10 days (Kempe, Silver, and O'Brien, 1970).

In the past, susceptibility to intercurrent infections frequently led to a fatal outcome. Modern supportive therapy and antibiotics have markedly improved the prognosis. Early and adequate penicillin therapy is curative, but, if diagnosis and treatment are delayed, irreversible renal damage may result.

\section{Conclusion}

On the basis of the findings in these 3 infants we suggest that the Treponema pallidum in congenital syphilis be added to the list of known or suspected antigens in human immune complex-induced glomerulonephritis. This list so far includes DNA antigen in disseminated lupus erythematosus nephritis (Koffler, Schur, and Kunkel, 1967), nephritogenic types of the Lancefield group A $\beta$-haemolytic-streptococcus in acute poststreptococcal glomerulonephritis (Michael et al., 1966), Plasmodium malariae in quartan malaria nephrotic syndrome (Ward and Kibukamusoke, 1969), coagulase negative and positive staphylococci in glomerulonephritis associated with infected ventriculoatrial shunts (Stickler et al., 1968), and penicillamine (Lachmann, 1968).

We thank Professor R. McDonald for constant encouragement and advice; Dr. H. Meyer, Acting Medical Superintendent, for permission to publish; M. v.d. Giessen from the Central Laboratory of the Red Cross Blood Transfusion Service, Amsterdam, for preparing the antisera; and Mrs. V. Eyles for preparation of the manuscript.

\section{REFERENCES}

Bhorade, M. S., Carag, H. B., Lee, H. J., Potter, E. V., and Dunea, G. (1971). Nephropathy of secondary syphilis. Fournal of the American Medical Association, 216, 1159. 


\section{Congenital syphilis and glomerulonephritis with evidence for immune pathogenesis}

Braunstein, G. D., Lewis, E. J., Galvanek, E. G., Hamilton, A., and Bell, W. R. (1970). The nephrotic syndrome associated with secondary syphilis. American fournal of Medicine, 48, 643.

Brentjens, J. R. H., Feltkamp-Vroom, T. M., Tiddens, H. A. W. M., and Kuyten, R. H. (1969). Localization of plasma proteins in kidneys of children with diffuse nephropathies, studied by an indirect immunofluorescent technique. Acta Paediatrica Scandinavica, 58, 374

Brophy, E. M., Ashworth, C. T., Arias, M., and Reynolds, J. (1964). Acute syphilitic nephrosis in pregnancy. Obstetrics and Gynecology, 24, 930.

Dixon, F. J. (1968). The pathogenesis of glomerulonephritis. American fournal of Medicine, 44, 493.

Dixon, F. J., and Cochrane, C. G. (1970). The pathogenicity of antigen-antibody complexes. In Pathology Annual, p. 355. Ed. by Sheldon C. Sommers. Appleton-Century-Crofts, New York.

Falls, W. F., Jr., Ford, K. L., Ashworth, C. T., and Carter, N. W. (1965). The nephrotic syndrome in secondary syphilis. Annals of Internal Medicine, 63, 1047.

Germuth, F. G., Jr., Senterfit, L. B., and Pollack, A. D. (1967). Immune complex disease. I. Experimental acute and chronic glomerulonephritis. Fohns Hopkins Medical fournal, 120, 225.

Herrmann, G., and Marr, W. L. (1935). Clinical syphilitic nephropathies. American fournal of Syphilis and Neurology, $19,1$.

Kempe, C. H., Silver, H. K., and O'Brien, D. (1970). (Editors) Current Pediatric Diagnosis and Treatment, p. 600. Lange Medical Publications, Los Altos, Calif.

Koffler, D., Schur, P. H., and Kunkel, H. G. (1967). Immunological studies concerning the nephritis of systemic lupus erythematosus. Fournal of Experimental Medicine, 126, 607.

Lachmann, P. J. (1968). Nephrotic syndrome from penicillamine. Postgraduate Medical fournal, 44. Suppl. on Penicillamine, 23.

Lewis, E. J., and Couser, W. G. (1971). The immunologic basis of human renal disease. Pediatric Clinics of North America, 18, 467.

McCluskey, R. T. (1970). Evidence for immunologic mechanisms in several forms of human glomerular diseases. Bulletin of the New York Academy of Medicine, 46, 769.

McDonald, R., Wiggelinkhuizen, J., and Kaschula, R. O. C. (1971). The nephrotic syndrome in very young infants. American fournal of Diseases of Children, 122, 507.

Michael, A. F., Drummond, K. N., Good, R. A., and Vernier, R. L. (1966). Acute poststreptococcal glomerulonephritis: immune deposit disease. Fournal of Clinical Investigation, 45, 237.

Mitchell, J. (1951). Congenital syphilis with acute nephritis. British Medical fournal, $1,396$.
Papaioannou, A. C., Asrow, G. G., and Schuckmell, N. H. (1961). Nephrotic syndrome in early infancy as a manifestation of congenital syphilis. Pediatrics, 27, 636.

Pollner, P. (1966). Nephrotic syndrome associated with congenital syphilis. Fournal of the American Medical Association, 198, 263.

Scully, J. P., and Yamazaki, J. N. (1949). Congenital syphilitic nephrosis successfully treated with penicillin. American fournal of Diseases of Children, 77, 652.

Stickler, G. B., Shin, M. H., Burke, E. C., Holley, K. E., Miller, R. H., and Segar, W. E. (1968). Diffuse glomerulonephritis with infected ventriculoatrial shunt. New England fournal of Medicine, 279, 1077.

Taitz, L. S., Isaacson, C., and Stein, H. (1961). Acute nephritis associated with congenital syphilis. British Medical fournal, $2,152$.

Ward, P. A., and Kibukamusoke, J. W. (1969). Evidence for soluble immune complexes in the pathogenesis of the glomerulonephritis of quartan malaria. Lancet, 1, 283.

Yampolsky, J., and Mullins, D. F., Jr. (1945). Acute glomerular nephritis in an infant with congenital syphilis. American Fournal of Diseases of Children, 69, 163.

Yuceoglu, A. M., Churg, J., Mallika, Y., and Pollner, P. (1966) Nephropathy due to congenital syphilis in a 10 week old infant. In Abstracts of the Third International Congress of Nephrology, Washington, D. C., 1966, p. 300.

Correspondence to Dr. Jan Wiggelinkhuizen, Red Cross War Memorial Children's Hospital, Rondebosch, Cape, South Africa.

\section{Addendum}

Since preparation of this paper two case reports have been published.

Kaplan, B. S., Wiglesworth, F. W., Marks, M. I., and Drummond, K. N. (1972). The glomerulopathy of congenital syphilis-an immune deposit disease. Journal of Pediatrics, 81, 1154.

Leighton Hill, L., Singer, D. B., Falletta, J., and Stasney, R. (1972). The nephrotic syndrome in congenital syphilis: an immunopathy. Pediatrics, 49, 260. 